\title{
Prospective Comparison of Angio-Seal versus Manual Compression for Hemostasis after Neurointerventional Procedures under Systemic Heparinization
}

\author{
H.-F. Wong, C.-W. Lee, Y.-L. Chen, Y.-M. Wu, H.-H. Weng, Y.-H. Wang, and H.-M. Liu
}

\begin{abstract}
BACKGROUND AND PURPOSE: The use of arterial closure device in patients with prolonged high ACT values has not been extensively studied. The aim of this study was to compare the safety and efficacy of an arterial closure device, Angio-Seal, with manual compression in patients on anticoagulation following neurointerventional procedures.
\end{abstract}

MATERIALS AND METHODS: This was a 2-center prospective study approved by our institutional review boards. In total, 153 consecutive patients with 174 arteriotomies (86 men, 67 women; mean age, $56.1 \pm 16.2$ years) following femoral arterial puncture for neurointerventional procedures were enrolled in convenience sampling. All of the patients were systemically heparinized with an ACT between 250 and 500 seconds before removal of the sheath after the procedure. Group 1 consisted of 104 arteriotomies that were closed with an arterial closure device (Angio-Seal), and group 2 consisted of 70 arteriotomies treated with manual compression. The ACT before sheath removal, time to hemostasis, and complications immediately and 24 hours after the procedure were recorded.

RESULTS: The mean ACT before sheath removal was $284.8 \pm 37.6$ seconds (range, 250-414 seconds). The mean hemostasis time was significantly shorter in group 1 ( $2.4 \pm 11.7$ minutes) compared with group 2 (44.7 \pm 27.4 minutes) $(95 \% \mathrm{Cl}, 38.16-51.24$ minutes; $P<.001)$. Hematoma occurred in 9 patients in group 1 (8.6\%) and 18 in group 2 (25.7\%). One patient developed an arterial occlusion after hemostasis with the closure device, but this was successfully revascularized.

CONCLUSIONS: Angio-Seal was found to safely and effectively achieve rapid closure of the femoral access site in patients undergoing neuroendovascular procedures under systemic heparinization with an ACT in the range of $250-500$ seconds.

ABBREVIATIONS: $\mathrm{ACT}=$ activated clotting time; $\mathrm{Cl}=$ confidence interval; $\mathrm{OR}=$ odds ratio

$\mathbf{T}$ he common femoral artery is the most frequently used site for vascular access in catheter-based neurointerventional procedures. Management of the femoral artery access site has become a more important consideration as the frequency of endovascular interventional procedures increases worldwide. Although manual compression has been accepted as the criterion standard for access-site hemostasis, it is limited by additional operator time, prolonged patient immobilization following the procedure, and

Received March 12, 2012; accepted after revision May 21

From the Department of Imaging and Intervention, Chang Gung Memorial Hospital at Linkou (H.-F.W., Y.-L.C., Y.-M.W.) and Chiayi (H.-H.W.), College of Medicine and School of Medical Technology, Chang-Gung University, Taiwan; and Department of Medical Imaging (C.-W.L., Y.-H.W., H.-M.L.), National Taiwan University Hospital, Taipei, Taiwan.

This work was supported by the National Central University, Taiwan (NSC 100-2911I-008-001) Center for Dynamical Biomarkers and Translational Medicine.

Please address correspondence to Hon-Man Liu, MD, Department of Medical Imaging, National Taiwan University Hospital, No. 7, Chung-Shan South Rd, Taipei,

Taiwan 10016; e-mail: hmliu@ntu.edu.tw

http://dx.doi.org/10.3174/ajnr.A3226 complication rates in the range of $1.3 \%-3.4 \% .{ }^{1-3}$ Additionally, hemostasis by manual compression in anticoagulated patients receiving larger access sheaths can be difficult to achieve. The major concern in achieving hemostasis of the arteriotomy after neurointerventional procedures is the level of anticoagulation, not only during the endovascular treatment but also after the procedure, when it occasionally is sustained to minimize thrombotic events. However, vascular closure device trials have rarely demonstrated lower complication rates compared with manual compression, and occasionally the use of vascular closure devices has resulted in complications primarily involving infection and thrombosis. These complications have become a significant source of litigation in many practices and hospital facilities.

The use of closure devices has been reported extensively in cardiology and, to a lesser extent, in interventional radiology. There are few publications regarding this subject in interventional neuroradiology. ${ }^{4-9}$ During the entire neurointerventional procedure, it is important to keep the ACT between 250 and $500 \mathrm{sec}-$ onds or 2 to 2.5 times that of baseline. This makes these patients at 
high risk of bleeding at the access site, especially following involuntary agitated movement during recovery from general anesthesia. Delayed sheath removal and sending the patient back to the intensive care unit have been common practices in interventional neuroradiology. Many serious complications such as a thromboembolic event, hematoma formation, retroperitoneal hemorrhage, or infection can occur when arterial sheaths are left in place after neurointerventional procedures. ${ }^{7,10}$

The use of a closure device has been shown to be effective in reducing the time to hemostasis and ambulation, subsequent to both transfemoral angiography and intervention. ${ }^{11-16}$ The AngioSeal VIP 6F/8F device (St. Jude Medical, Minnetonka, Minnesota) is a vascular access closure device that achieves hemostasis by compressing the arteriotomy site between a bioabsorbable intravascular foot plate and an external bovine collagen sponge. The anchor is resorbed physically within 30 days and chemically within 90 days. The use of Angio-Seal in patients with prolonged high ACT values has not been extensively studied, to our knowledge. In this prospective study, we aimed to compare Angio-Seal with manual compression in patients with prolonged high values of ACT just before arterial sheath removal.

\section{MATERIALS AND METHODS}

Following local institutional review board approval and written informed consent from all study subjects, 153 consecutive patients ( 86 men, 67 women; mean age, $56.1 \pm 16.2$ years) were prospectively divided by convenience sampling into 2 groups. $\mathrm{Pa}-$ tients were enrolled into the study at Chang Gung Memorial Hospital (Linkou Medical Center) and National Taiwan University Hospital between March 8, 2010, and February 8, 2011. Following an explanation of the risks and benefits of the options, all patients themselves chose either the use of an arterial closure device (Angio-Seal) or manual compression for hemostasis of the arteriotomy before entering our study. All vascular access was obtained via the common femoral artery. At the end of the neurointerventional procedure, ACT was checked before access-sheath removal. Patients in group 1 received hemostasis by using the arterial closure device (Angio-Seal VIP 6F/8F), and patients in group 2 received manual compression. A certified interventional neuroradiologist performed all procedures. All operators had experience of $>50$ successful Angio-Seal deployments. The deployment of the device and the application of manual compression were performed by the same operator who performed the neurointerventional procedures. ACT before sheath removal, time to hemostasis, and complications occurring immediately and 24 hours after the procedure were recorded.

The inclusion criteria were as follows: 1) Patients received an endovascular neurointerventional procedure, 2) ACT was within the range of 250-500 seconds after the procedure and before sheath withdrawal, and 3) puncture was in the common femoral artery (this was confirmed angiographically before sheath withdrawal). The exclusion criteria were the following: 1) patients who were pregnant or lactating, 2) patients younger than 18 years of age, 3) patients with a bleeding disorder, and 4) groin infection.

In group 1, a $6 \mathrm{~F}$ Angio-Seal was used in those patients with a $5 \mathrm{~F}$ or $6 \mathrm{~F}$ access sheath, and an $8 \mathrm{~F}$ Angio-Seal was used for those with a $7 \mathrm{~F}$ or $8 \mathrm{~F}$ access sheath. In both groups, after complete hemosta- sis had been obtained, patients' pedal pulses were evaluated to check their blood flow. In both groups, if complete hemostasis could not be obtained after 30 minutes, a hemostasis-enhancing pad, Clo-Sur PAD (Scion Cardio-Vascular, Miami, Florida), was used to provoke hemostasis for ethical reasons.

Patients were monitored for subsequent complications for 20-24 hours. The hematoma was inspected grossly and measured with a ruler by our assistant operator 24 hours later. Size was determined as the longest dimension measured across the access site. Evaluation after faculty discussion was performed if the operator encountered a controversial hematoma of $10 \mathrm{~cm}$. Complications were defined as minor and major. Minor complications included a local hematoma of $<10 \mathrm{~cm}$ in diameter. Major complications were those requiring further percutaneous or surgical intervention, as well as a local hematoma of $>10 \mathrm{~cm}$ in diameter, ${ }^{17}$ pseudoaneurysm, groin infection, retroperitoneal bleeding, arteriovenous fistula, bleeding at the site requiring transfusion, device embolization, and death. In addition, hemostasis time, the ACT value, and the puncture technique (single- or double-wall puncture) were also recorded.

The stratification of ACT levels was arbitrary, based on every 50 -second increase. The patient numbers above 350 seconds were summed. The stratification of the ACT was treated as an exposure variable in the stage of data analysis. We treated 2 arteriotomies in the same patient as separate and independent events. The numeric variables were presented as mean $\pm \mathrm{SD}$. We used logistic regression analysis to identify factors linked with outcomes. Multivariate logistic regression was used to estimate the association between Angio-Seal use and the severity of ACT prolongation and the risk of hematoma development. In the analysis, the subjects were categorized into 1 of the 2 categories: manual compression versus Angio-Seal usage. ORs and their 95\% CIs were calculated by using the lowest ACT group (250-300) as the reference group. The association between Angio-Seal usage and hematoma occurrence was stratified by ACT values. All statistical analyses were performed by using STATA, Version 11.0 statistical software (StataCorp, College Station, Texas). A $P$ value $\leq .05$ indicated a statistically significant difference.

\section{RESULTS}

A total of 174 arteriotomies in 153 patients were included in our analysis. The neurointerventional treatment indication included cerebral aneurysms $(n=48,31.4 \%)$, arteriovenous fistulas ( $n=$ $31,20.3 \%)$, vascular malformations $(n=9,5.8 \%)$, intra-/extracranial stenosis $(n=55,36.9 \%)$, and preoperative tumor embolization $(n=10,6.5 \%)$.

There were 104 arteriotomies in group 1 and 70 arteriotomies in group 2.

The overall mean ACT was $284.8 \pm 37.7$ seconds (range, $250-$ 414 seconds). The mean ACT of group 1 was $287.9 \pm 40.5$ seconds (range, 250-414 seconds). The mean ACT of group 2 was $280.2 \pm$ 32.8 seconds (range, 250-374 seconds). All of the Angio-Seal devices were deployed successfully. The mean hemostasis time was $2.4 \pm 11.8$ minutes in group 1 compared with $44.7 \pm 27.4$ minutes in group 2 . The 2 -sample $t$ test showed a significantly shorter hemostasis time $(P<.001)$ in group 1 compared with group 2 . In addition, the Clo-Sur PAD was used to provoke hemostasis if 
Table 1: Complication rates and a comparison of hematoma rates between Angio-Seal and manual compression at different ACT levels ${ }^{a}$

\begin{tabular}{|c|c|c|c|c|c|c|c|c|c|c|}
\hline \multirow[b]{3}{*}{$\mathrm{ACT}(\mathrm{sec})$} & \multicolumn{5}{|c|}{ Angio-Seal } & \multicolumn{5}{|c|}{ Manual Compression } \\
\hline & \multirow{2}{*}{$\begin{array}{l}\text { Without } \\
\text { Hematoma }\end{array}$} & \multicolumn{3}{|c|}{ With Hematoma } & \multirow{2}{*}{$\begin{array}{c}\text { Total } 104 \\
(90)^{\mathrm{b}}\end{array}$} & \multirow{2}{*}{$\begin{array}{l}\text { Without } \\
\text { Hematoma }\end{array}$} & \multicolumn{3}{|c|}{ With Hematoma } & \multirow{2}{*}{$\begin{array}{c}\text { Total } 70 \\
(67)^{\mathrm{b}}\end{array}$} \\
\hline & & $<3 \mathrm{~cm}$ & $3-6 \mathrm{~cm}$ & $>10 \mathrm{~cm}$ & & & $<3 \mathrm{~cm}$ & $3-6 \mathrm{~cm}$ & $>10 \mathrm{~cm}$ & \\
\hline $250-300$ & $72(61)$ & $1(1)$ & & & $73(62)$ & $43(43)$ & $4(4)$ & $6(6)$ & $3(2)$ & $56(55)$ \\
\hline $300-350$ & $14(12)$ & $4(4)$ & & $1(1)$ & 19 (17) & $6(4)$ & & $3(3)$ & & $9(7)$ \\
\hline $350-500$ & $9(9)$ & $3(2)^{c}$ & & & $12(11)$ & $3(3)$ & & $2(2)$ & & $5(5)$ \\
\hline
\end{tabular}

${ }^{a}$ Complication rate (hematoma $\left.>10 \mathrm{~cm}\right) 0.96(1.11) \%$ for Angio-Seal and $4.28(2.98) \%$ for manual compression. Patient numbers and complication rates in parentheses.

${ }^{b}$ Four patients with bilateral arteriotomies closed by Angio-Seal and manual compression, respectively.

c One patient with bilateral Angio-Seal and bilateral hematomas simultaneously.

Table 2: Results of uni- and multivariate logistic regression analysis derived from hematoma development and independent predictors

\begin{tabular}{|c|c|c|c|c|c|c|}
\hline \multirow[b]{2}{*}{ Variable } & \multicolumn{3}{|c|}{ Univariate } & \multicolumn{3}{|c|}{ Multivariate } \\
\hline & OR & $95 \% \mathrm{Cl}^{\mathrm{a}}$ & $P$ Value & OR & $95 \% \mathrm{Cl}^{\mathrm{a}}$ & $P$ Value \\
\hline \multicolumn{7}{|l|}{ ACT time (sec) } \\
\hline $250-300$ & 1 (baseline) & & & 1 (baseline) & & \\
\hline $300-350$ & 3.3 & $1.2-8.8$ & $.019^{\mathrm{b}}$ & 4.5 & $1.5-13.2$ & $.006^{\mathrm{b}}$ \\
\hline $350-500$ & 3.4 & $1.1-11.2$ & $.041^{\mathrm{b}}$ & 5.0 & $1.4-18.0$ & $.015^{b}$ \\
\hline Angio-Seal use & 0.3 & $0.1-0.7$ & $.003^{b}$ & 0.2 & $0.1-0.5$ & $.001^{\mathrm{b}}$ \\
\hline Age & 1.0 & $1.00-1.02$ & .928 & & & \\
\hline Sex & 1.4 & $0.6-3.2$ & .426 & & & \\
\hline Side & 0.99 & $0.3-3.1$ & .984 & & & \\
\hline Puncture technique (single- vs double-wall) & 1.2 & $0.6-2.3$ & .658 & & & \\
\hline
\end{tabular}

${ }^{a}$ Confidence interval is adjusted for age, sex, and puncture methods.

b Significant difference from the control $(P<.05)$

initial hemostasis was not complete after 30 minutes. There were 49 patients (51 arteriotomies) who required Clo-Sur PAD exclusively in the group with manual compression.

In total, hematoma occurred in 27 arteriotomies/26 patients (15.5\%/16.9\%): Nine arteriotomies/8 patients in group $1(8.6 \% /$ $9.2 \%)$ and 18 arteriotomies/18 patients in group 2 (25.7\%). However a major complication with a hematoma of $>10 \mathrm{~cm}$ occurred 1 of 87 patients in group $1(1.1 \%)$ and in 3 of 70 patients in group $2(4.3 \%)$, with a significant difference in favor of group $1(\mathrm{OR}=$ 0.3 ; 95\% CI, $0.1-0.7 ; P=.003$ ) (Table 1 ). In group 1 , only 1 patient had a hematoma of $>3 \mathrm{~cm}$. This patient received multiple punctures in a single common femoral artery. Bleeding resulted from the puncture site where the Angio-Seal had not been deployed, and it was stopped in 2 hours after application of a CloSur PAD device. This patient developed a right inguinal ecchymosis and a $10-\mathrm{cm}$ hematoma. In group 2, eighteen hematomas occurred, 14 of which were $>3 \mathrm{~cm}$ in diameter.

Fourteen hematomas developed in 129 patients with ACT in the range of $250-300$ seconds: One $(1 / 73=1.4 \%)$ occurred in group 1 and $13(13 / 56=23.2 \%)$ occurred in group 2. Eight hematomas developed in 28 patients with an ACT in the range of $300-350$ seconds: Five $(5 / 19=26.3 \%)$ occurred in group 1 and 3 $(3 / 9=33.3 \%)$ in group 2 . Five hematomas developed in 17 patients with ACT in the range of 350-500 seconds: Three $(3 / 12=$ $25 \%)$ occurred in group 1 , and $2(2 / 5=40 \%)$, in group 2 (Table 1). The adjusted ORs (95\% CIs) were 4.5 (1.5-13.2) for the patients with ACT values between 300 and 350 and 5.0 (1.4-18.0) for the patients with ACT values between 350 and 500 compared with those with the lowest ACT values (250-300) (Table 2). The adjusted OR (95\% CI) was $0.2(0.1-0.5)$ for group 1 compared with group 2.

In achieving vascular access, 125 patients underwent singlewall puncture and 49 patients underwent double-wall puncture. There were 76 patients with single-wall punctures $(60.8 \%)$ and 28

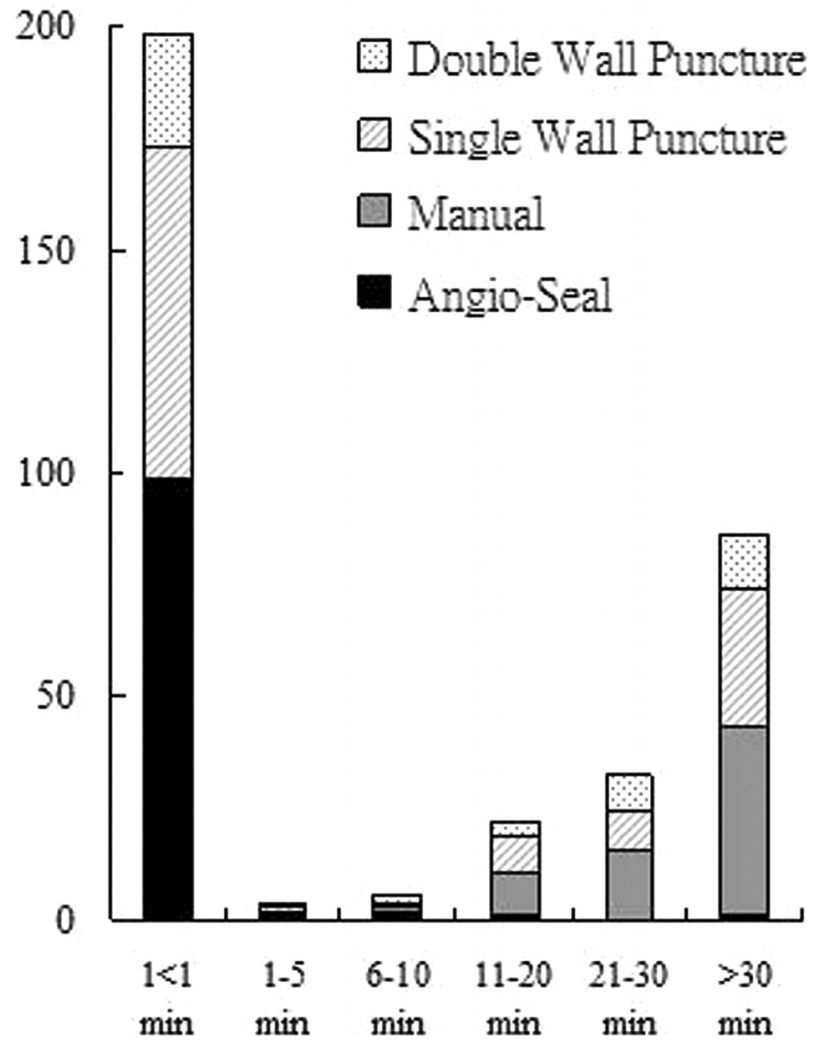

FIG 1. Comparison of hemostasis times for the number of patients receiving Angio-Seal versus manual compression, including a comparison of puncture techniques.

with double-wall punctures (57.1\%) in group 1 and 49 patients with single-wall punctures (39.2\%) and 21 with double-wall punctures $(42.9 \%)$ in group 2 . There was no significant difference between the 2 puncture methods ( $\mathrm{OR}=1.2, P=.658$ ) (Fig 1 ).

One major complication occurred in group 1. A 75-year-old

AJNR Am J Neuroradiol 34:397-401 Feb 2013 www.ajnr.org

399 

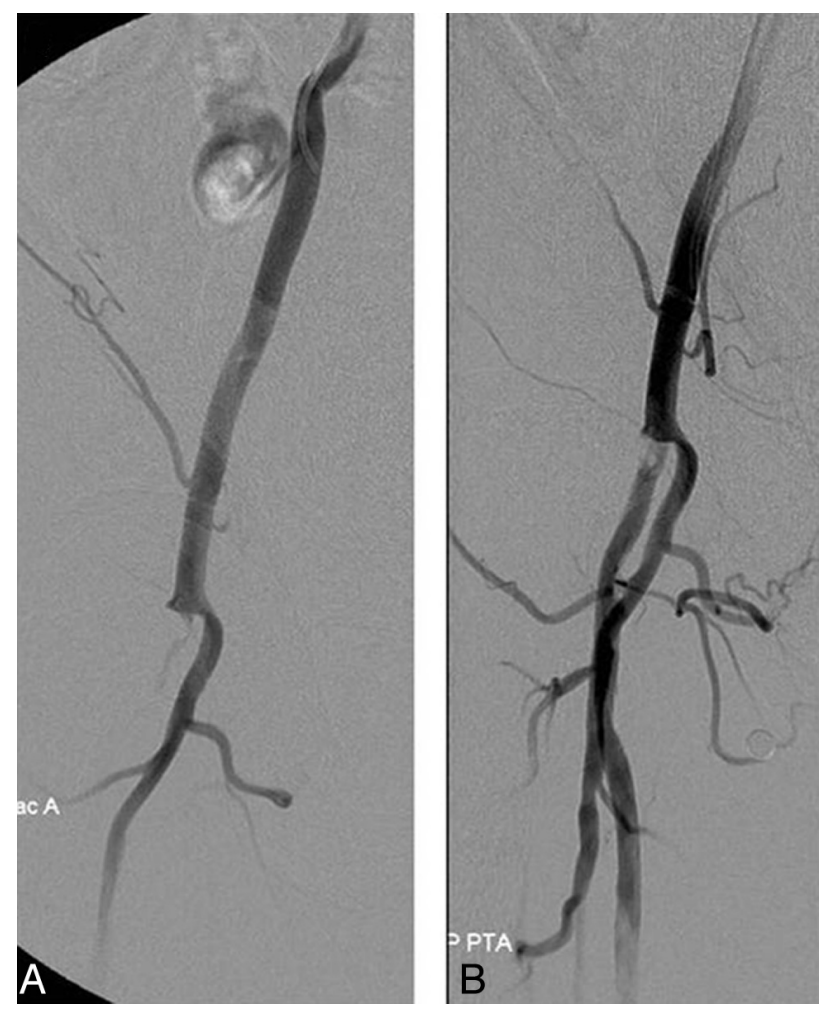

FIG 2. Right common iliac artery angiogram showing occlusion at the common femoral artery $(A)$ and revascularization after angioplasty $(B)$.

man had undergone carotid angioplasty and stent placement for a symptomatic high-grade stenosis of the carotid artery. No obvious local abnormality was shown on the angiogram before deployment of the closure device, but the pedal pulse was found to have diminished a few hours later. An angiogram showed an occluded common femoral artery at the puncture site. Flow was restored following balloon angioplasty (Fig 2). Anticoagulation was maintained for 24 hours, and no change in flow was apparent by sonography and during clinical follow-up. Despite the complication, there was no delay in the discharge of this patient.

No new complications or progression of hematoma were found in either group at the 24-hour follow-up.

\section{DISCUSSION}

In our study, we found that patients treated with Angio-Seal more rapidly achieved hemostasis than those treated with manual compression, with less time needed for hemostasis, less local hematoma formation, and an acceptably low level of complications in patients with prolonged elevated ACT after endovascular neurointerventional procedures. The Angio-Seal device is an alternative hemostatic technique that can replace the labor-intensive method of manual compression and may avoid repetitive stress injury. ${ }^{18}$ In our study, there were no deployment failures and the complication rate was low. Despite 1 adverse event in group 1, there were no delays in patient discharge. In a study of 698 Angio-Seal closures, the minor complication rate in the interventional group was $2.4 \%$ and the major complication rate in this group was $1.4 \% .{ }^{4}$ This was higher than that in our group. In a study of control of bleeding after transfemoral catheterization, the mean time to hemostasis with manual compression was 33.5 minutes in a multicenter trial. ${ }^{19}$ For the manual compression group in our study, the mean time to hemostasis was 44.7 minutes, but we found that it was necessary to use the Clo-Sur PAD in 42 patients $(60 \%)$, when the time of hemostasis was $>30$ minutes. Therefore the actual hemostasis time in our study would have been longer if only manual compression had been used. Our higher values probably result from the fact that all patients in our cohort were on an anticoagulation regimen during the procedure with definitely prolonged elevated ACT values.

Khaghany et $\mathrm{al}^{5}$ reported overall complication rates of $0.6 \%$ in their device (Perclose; Abbott Vascular, Santa Clara, California) group and $2.5 \%$ in their manual compression group $(P=.15)$. McTaggart et $\mathrm{al}^{6}$ reported a rate of groin hematomas of $1.4 \%$ in their StarClose Vascular Closure System (Abbott Vascular) group and $4.2 \%$ in their manual compression group for the patients not suited for StarClose deployment. McTaggart et al reported that the rate of groin hematomas was $4.2 \%$ in the manual compression and $1.4 \%$ in StarClose vascular closure group. ${ }^{6}$ To the best of our knowledge, there have been no prospective studies that have compared Angio-Seal with manual compression in neurointerventional patients with reference to ACT values. Our study shows that an increase in ACT results in a greater frequency of hematoma formation. Overall, we found no significant difference in hematoma formation between single-wall and double-wall punctures, though there was a slight increase in the manual compression group. In a meta-analysis of 6 studies that compared AngioSeal with manual compression, the random-effects analysis demonstrated an OR of 0.86 (95\% CI, 0.51-1.45; $P=.78$ ) for hematoma events and an OR of 0.30 (95\% CI, 0.04-2.07; $P=.93$ ) for pseudoaneurysms. Both marginally favor a trend toward Angio-Seal having fewer complications than manual compression. ${ }^{20}$ Our study confirms a similar result in favor of the Angio-Seal device even in patients with prolonged elevated ACT values.

There are limitations in our study. One of the advantages of achieving rapid hemostasis when using a closure device is the potential for early ambulation of the patient. We were unable to evaluate the effect of Angio-Seal on ambulation time because most of our patients had to stay in the intensive care unit for at least 1 night. In addition, many, if not most, of the Angio-Sealrelated complications may have occurred after 24 hours, so we would not have found them. Most of our ACT values were within the range of 250-300 seconds. A larger scale randomized controlled trial in interventional neuroradiology is warranted to evaluate the safety and efficacy of Angio-Seal use in patients with prolonged higher ACT values.

\section{CONCLUSIONS}

Groin hematomas appear to be less frequent when Angio-Seal is used compared with manual compression, particularly in cases when the ACT value is prolonged at an elevated level. Angio-Seal is rapid, safe, and effective in achieving closure of the femoral access site in patients undergoing neuroendovascular procedures under systemic heparinization with their postprocedure ACT ranging from 250 to 500 seconds. 


\section{REFERENCES}

1. Bogart DB, Bogart MA, Miller JT, et al. Femoral artery catheterization complications: a study of 503 consecutive patients. Cathet Cardiovasc Diagn 1995;34:8-13

2. Heintzen MP, Strauer BE. Peripheral arterial complications after heart catheterization [in German]. Herz 1998;23:4-20

3. Pracyk JB, Wall TC, Longabaugh JP, et al. A randomized trial of vascular hemostasis techniques to reduce femoral vascular complications after coronary intervention. Am J Cardiol 1998;81:970-76

4. Geyik S, Yavuz K, Akgoz A, et al. The safety and efficacy of the AngioSeal closure device in diagnostic and interventional neuroangiography setting: a single-center experience with 1,443 closures. $\mathrm{Neu}$ roradiology 2007;49:739-46

5. Khaghany K, Al-Ali F, Spigelmoyer T, et al. Efficacy and safety of the Perclose Closer $S$ device after neurointerventional procedures: prospective study and literature review. AJNR Am J Neuroradiol 2005;26:1420-24

6. McTaggart RA, Raghavan D, Haas RA, et al. StarClose vascular closure device: safety and efficacy of deployment and reaccess in a neurointerventional radiology service. AJNR Am J Neuroradiol 2010;31:1148-50

7. Morris PP, Braden G. Neurointerventional experience with an arteriotomy suture device. AJNR Am J Neuroradiol 1999;20:1706-09

8. Park Y, Roh HG, Choo SW, et al. Prospective comparison of collagen plug (Angio-Seal) and suture-mediated (the Closer S) closure devices at femoral access sites. Korean J Radiol 2005;6:248-55

9. Pierot L, Herbreteau D, Bracard S, et al. An evaluation of immediate sheath removal and use of the Angio-Seal vascular closure device in neuroradiological interventions. Neuroradiology 2006;48:45-49

10. Koenigsberg RA, Wysoki M, Weiss J, et al. Risk of clot formation in femoral arterial sheaths maintained overnight for neuroangiographic procedures. AJNR Am J Neuroradiol 1999;20:297-99

11. Cremonesi A, Castriota F, Tarantino F, et al. Femoral arterial hemostasis using the Angio-Seal ${ }^{\mathrm{a}}$ system after coronary and vascular per- cutaneous angioplasty and stenting. J Invasive Cardiol 1998;10: 464-69

12. Kapadia SR, Raymond R, Knopf W, et al. The 6Fr Angio-Seal arterial closure device: results from a multimember prospective registry. Am J Cardiol 2001;87:789-91, A8

13. Kussmaul WG 3rd, Buchbinder M, Whitlow PL, et al. Rapid arterial hemostasis and decreased access site complications after cardiac catheterization and angioplasty: results of a randomized trial of a novel hemostatic device. J Am Coll Cardiol 1995;25:1685-92

14. Sanborn TA, Gibbs HH, Brinker JA, et al. A multicenter randomized trial comparing a percutaneous collagen hemostasis device with conventional manual compression after diagnostic angiography and angioplasty. J Invasive Cardiol 1999;11(suppl B):6B-13B

15. Schrader R, Steinbacher S, Burger W, et al. Collagen application for sealing of arterial puncture sites in comparison to pressure dressing: a randomized trial. J Invasive Cardiol 1999;11(suppl B):14B-18B

16. Silber S, Bjorvik A, Muhling $\mathrm{H}$, et al. Usefulness of collagen plugging with VasoSeal after PTCA as compared to manual compression with identical sheath dwell times. Cathet Cardiovasc Diagn 1998;43:421-27

17. Tavris DR, Gallauresi BA, Dey S, et al. Risk of local adverse events by gender following cardiac catheterization. Pharmacoepidemiol Drug Saf 2007;16:125-31

18. Walker SB, Cleary S, Higgins M. Comparison of the FemoStop device and manual pressure in reducing groin puncture site complications following coronary angioplasty and coronary stent placement. Int J Nurs Pract 2001;7:366-75

19. Semler HJ. Transfemoral catheterization: mechanical versus manual control of bleeding. Radiology 1985;154:234-35

20. Das R, Ahmed K, Athanasiou T, et al. Arterial closure devices versus manual compression for femoral haemostasis in interventional radiological procedures: a systematic review and meta-analysis. Cardiovasc Intervent Radiol 2011;34:723-38 